\title{
PHOSPHORYLATION OF $d$-GLUCOSAMINE BY BRAIN EXTRACTS
}

\begin{abstract}
By R. P. HARPUR* and Prof. J. H. QUASTEL, F.R.S.
Montreal General Hospital Research institute, Montreal

$\mathrm{D}$ URING an iny estigation into the relationship between acetylcholine synthesis and carbohydrate metabofism in the central nervous system, it became important for us to study the inhibitory action of ghacose and fructose on the rate of acetylcholine formation by extracts of acetone-dried brain powder. Acetylcholine synthesis in such extracts and in homogenates proceeds rapidly under anaerobic conditions provided adenosinetriphosphate is present ${ }^{1,2}$. Feldberg and Mann ${ }^{1}$ also reported an inhibitory action of hexoses on this process. They made the suggestion that the sugars exercised their effects by being phosphorylated at the expense of adenosinetriphosphate, the quantity of the latter thus being diminished and the rate of acetylcholine synthesis being correspondingly decreased.

Using the manometric technique of Colowick and Kalckar ${ }^{3}$, we have been able to show that glucose and fructose are phosphorylated at the expense of adenosinetriphosphate in the presence of the brain extracts and to correlate this reaction with the

tube. Experiments were conducted at $27^{\circ}$ C., and adenosinetriphosphate (final concentration $0.002 M$ ) was tipped into the main vessel after twenty minutes equilibration and gassing with 93 per cent nitrogen : 7 per cent carbon dioxide. The final volume of fluid in the vessel was $3.0 \mathrm{ml}$. The rate of gas (carbon dioxide) output was measured in the conventional manner; the rate is proportional to the speed of the reaction between hexose or glucosamine and adenosinetriphosphate. Two commercial preparations of glucosamine hydrochloride and one of the free base* were used; all were found to give the same results. Control experiments which contained no sugar were always run, and the figures from these controls have been subtracted from the experimental figures to make a correction for the small adenosinetriphosphatase activity of the extracts and the change in gas volume when adenosinetriphosphate is tipped into the main vessel. It should be mentioned that, in the absence of adenosinetriphosphate, no gas output is observed from the sugars or $d$-glucosamine.
\end{abstract} inhibition of acetylcholine synthesis. While this work was in progress, Wiebelhaus and Lardy ${ }^{4}$ reported observations on the phosphorylation of glucose and fructose by acetone powder extracts of beef brain. They showed also that glucose has a higher affinity than fructose for the phosphorylating system. Meyerhof and his co-workers ${ }^{5,6,7}$ have carried out extensive studies on brain homogenates which show that glycolytic rates and hexokinase activities for glucose, and particularly for fructose, are dependent upon adenosinetriphosphate and sugar concentrations.

We now wish to report that $d$-glucosamine is phosphorylated at the expense of adenosinetriphosphate in the presence of extracts of acetone-dried brain and that, like glucose and fructose, it also produces an inhibition of the rate of acetylcholine synthesis in these extracts. Further details of this inhibition will be given in a later publication". Relatively little is known of the metabolism of this important aminosugar, and the finding that it may be phosphorylated, according to manometric studies, by adenosinetri. phosphate should stimulate further work on the subject. The finding is also of interest in view of the recent report of Jacobs ${ }^{9}$ that serum glucosaminelevels are high in diabetes mellitus and that the levels are reduced during insulin treatment.

Beef brains were obtained from the abattoirs within thirty minutes of the death of the animals, and the grey matter was removed and ground in ice-cold acetone. After filtering, the resulting powder was stored in a vacuum desiccator in the refrigerator. The extracts were made with water and centrifuged. The somewhat opalescent centrifugate was used for phosphorylation studies. A quantity of centrifugate, corresponding to the extract of $45 \mathrm{mgm}$. of dry powder, was placed in a Warburg manometer vessel which contained a solution of salts giving a final concentration of $0.028 M \mathrm{NaHCO}_{3}, 0.028 M \mathrm{KF}$, $0.004 M \mathrm{MgSO}_{4}$. The hexose or $d$-glucosamine was added at an appropriate concentration and sodium adenosinetriphosphate solution was placed in the side

* Canada Packer Research Fellow (McGill University).

\section{Phosphorylation of d-Glucosamine and Hexoses}

Typical rates of gas output by glucose, fructose and $d$-glucosamine in the presence of $0.002 M$ adenosinetriphosphate, under the experimental conditions described, are shown in Table 1. It will be

Table 1

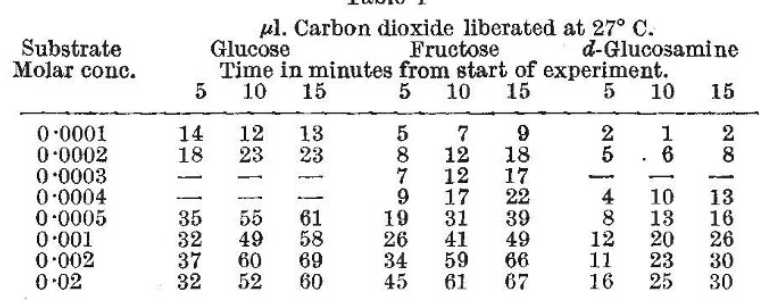

noted that with low concentrations of glucose, where the adenosinetriphosphate concentration is in no way limiting, the gas outputs in the first fifteen minutes approach the theoretical value for the conversion of glucose to hexosediphosphate. As pointed out by Wiebelhaus and Lardy ${ }^{4}$, this makes the determination of $K_{M}$ difficult by the manometric method. The maximum velocity of phosphorylation takes place at a lower concentration of glucose than that of fructose, while the speed of phosphorylation of $d$-glucosamine is appreciably less than that of fructose. Nevertheless, kinetic studies make it clear that the affinity of $d$-glucosamine to the enzyme involved is of the same order as that of fructose. Approximate values for the Michaelis constants are as follows: glucose $K_{M}=0.0001$, fructose $K_{M}=$ $0 \cdot 0007, d$-glucosamine $K_{M}=0.0006$.

\section{Competition Between d-Glucosamine and Hexoses}

Experiments with mixtures of the hexoses and $d$-glucosamine at appropriate concentrations indicate that no addition of the rates of phosphorylation

* $d$-(+)Glucosamine free base was kindly supplied by Ciba Co, Ltd. (Montreal). 


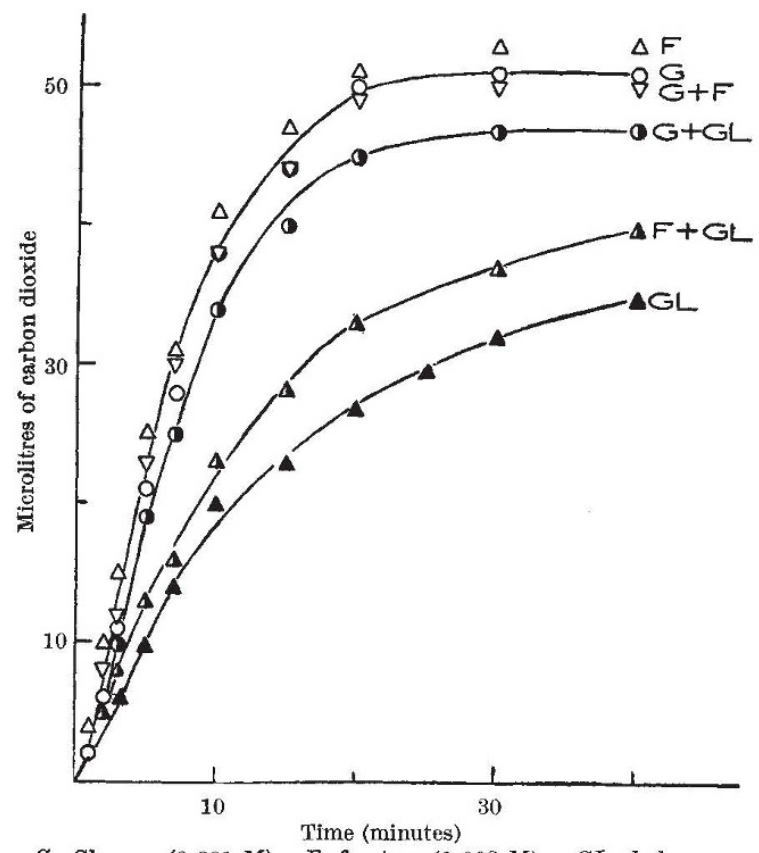

$G$, Glucose $(0.001 M) ; F$, fructose $(0.002 M) ; G L, d$-glucosamine $(0.002 M) ; G+F$, glucose $(0.001 M)+$ fructose $(0.002 M) ; \quad G+G L$ glucose $(0.001 M)+d$-glucosamine $(0.002 M) ; \quad F+G L$, fructose $(0.002 M)+d$-glucosamine $(0.002 M)$

occurs, and the results are consistent with a competition of all three substrates for the enzyme involved. The data shown in the accompanying graph bear out this conclusion. No correction, however, has been made for the acid-binding power of $d$-glucosamine. While there is only a slight inhibitory effect, if any, of $d$-glucosamine on glucose phosphorylation at the concentrations given, the presence of $d$-glucosamine reduces the rate of gas output due to fructose by far more than can be accounted for by acid-binding. It is evident that competition between all three substrates does occur, that between fructose and $d$-glucosamine being the most apparent. This would be expected from the fact that the affinities of these two molecules for the phosphorylating enzyme are of the same order.

\section{Competitive Inhibition by N-Acetylglucosamine}

The inhibition by $d$-glucosamine of hexose phosphorylation by adenosinetriphosphate might be interpreted as partly, if not wholly, due to competition not for a common enzyme but for the adenosinetriphosphate itself. This explanation is made unlikely by our finding that $\mathrm{N}$-acetylglucosamine, which is not phosphorylated, since there is no gas output with adenosinetriphosphate under the experimental conditions described, exercises a large

Table 2

$\begin{array}{ccc}\mu l & \mu \text {. Carbon dioxide liberated at } 27^{\circ} \mathrm{C} \text {. } \\ \text { Molar conc. } & \text { Glucose } & \text { Fructose }\end{array}$

N-acetyl

glucosamin

$$
\begin{array}{ccc}
\text { Glucose } & \text { Fructose } & d \text {-Glucosamin } \\
0.001 M & 0.002 M & 0.002 M
\end{array}
$$

\begin{tabular}{|c|c|c|c|c|c|c|c|c|c|}
\hline \multirow{2}{*}{ glucosamine } & \multicolumn{9}{|c|}{ Time in minutes from start of experiment } \\
\hline & 5 & 10 & 15 & 5 & 10 & 15 & 5 & 10 & 15 \\
\hline 0 & 32 & 49 & 58 & 28 & 48 & 56 & 10 & 19 & 26 \\
\hline $0 \cdot 001$ & - & - & - & 9 & 16 & 21 & 9 & 15 & 21 \\
\hline 0.002 & - & $\ldots$ & 一 & 2 & 6 & 8 & 3 & 8 & 12 \\
\hline 0.003 & $\longrightarrow$ & - & $\rightarrow$ & 4 & 7 & 8 & 3 & 9 & 12 \\
\hline 0.004 & - & - & - & 3 & 5 & 5 & 3 & 7 & 9 \\
\hline 0.008 & 20 & 35 & 45 & 1 & 4 & 4 & $\longrightarrow$ & $\longrightarrow$ & 一 \\
\hline
\end{tabular}

inhibitory action on the rates of phosphorylation of fructose and of $d$-glucosamine. Typical results showing the gas outputs obtained with mixtures of $\mathrm{N}$-acetylglucosamine, at difierent concentrations, and glucose, fructose or $d$-glucosamine are shown in Table 2. It will be noted that $\mathrm{N}$-acetylglucosamine brings about a relatively large inhibition of the rate of phosphorylation of fructose, the percentage inhibition being greater than that obtained with $d$-glucosamine. Its inhibitory effect on the phosphorylation of glucose is only perceptible at relatively high concentrations.

These results are all consistent with the view that $\mathrm{N}$-acetylglucosamine has an affinity for the enzyme involved in the phosphorylation of glucose, fructose and $d$-glucosamine. It seems strange that the enzyme which phosphorylates fructose at the expense of adenosinetriphosphate should also bring about a phosphorylation of $d$-glucosamine. Yet the inhibitory effects of $\mathrm{N}$-acetylglucosamine on both processes make it difficult to believe that is not the case. The effects cannot be due to the $N$-acetyl group alone since acetylglycine has no adverse effect upon the phosphorylation of $d$-glucosamine.

Summarizing these results, it is evident that extracts of brain bring about a phosphorylation of $d$-glucosamine at the expense of adenosinetriphosphate, that glucose, fructose and $d$-glucosamine all compete for the phosphorylase involved, glucose having the highest affinity, and that $\mathrm{N}$-acetylglucosamine, which is not phosphorylated, acts as a competitive inhibitor to all three substrates.

${ }^{2}$ Feldberg, W., and Mann, T., J. Physiol., 104, 8 (1945-6).

${ }^{2}$ Nachmansohn, D., and Machado, A. L., J. Neurophysiol., 6, 397 (1943).

${ }^{3}$ Colowick, S. P., and Kalckar, H. M., J. Biol. Chem., 148, 117 (1943).

'Wiebelhaus, V. D., and Lardy, H. A., Archiv. Biochem., 21, 321 (1949).

${ }^{5}$ Meyerhof, 0., and Geliazkowa, Nevena, Archiv. Biochem., 12, 405 (1947).

- Meverhof, O., Archiv. Biochem., 13, 485 (1947).

'Meyerhof, O., and Wilson, Jean R., Archiv. Biochem., 19, 502 (1948). - Harpur, R. P., and Quastel, J. H., Nature (in the press).

'Jacobs, H. R., J. Lab. Clin. Med., 34, 116 (1949).

\section{QUANTITATIVE ESTIMATION OF MARINE NANNOPLANKTON \\ By H. A. COLE and E. W. KNIGHT JONES}

Ministry of Agricylture and Fisheries, Conway, N. Wales

A $\mathrm{T}$ a recent meeting of the Challenger Society our attention was directed to the need for a restatement of the technique for the estimation of nannoplankton flagellates and algæ. Appreciation of the imporfance of these very small forms as food for marine larvæ and filter feeders is growing, although little is known as yet of their distribution in the sea or of their seasonal variation in abundance.

The method successfully employed at Conway for the past fifteen years ${ }^{1}$ is based upon the concentration of a known volume of sea water by filtration through a collodion membrane. The filtering apparatus used was formerly made in Germany; but Messrs. Baird and Tatlock, Ltd., have recently manufactured an improved model under the trade name 'Stefi'. The apparatus (see figure) comprises a cylindrical glass container (a) with an open ground bottom, which rests on the collodion membrane $(b)$, which, in its turn, is separated from a sintered glass plate by a filter paper (c) (Whatman No. 3 is very suitable). 